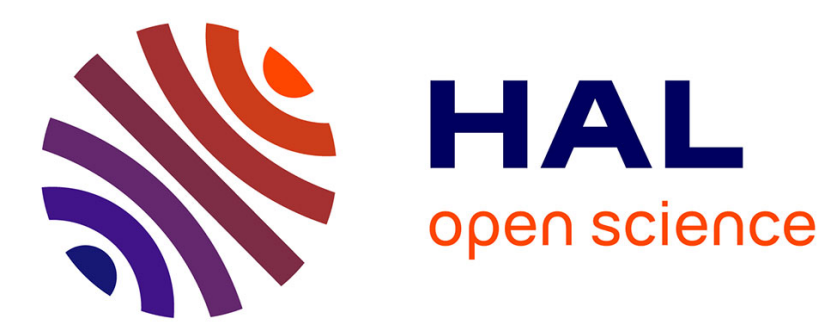

\title{
Overall behaviour of a truss of fibers linked by viscous joints
}

\author{
Steven Le Corre, Denis Caillerie, Denis Favier, Laurent Orgéas
}

\section{To cite this version:}

Steven Le Corre, Denis Caillerie, Denis Favier, Laurent Orgéas. Overall behaviour of a truss of fibers linked by viscous joints. Composites for the millenium (FRC2000), 2000, Newcastle-upon-Tyne, United Kingdom. pp.303 - 310, 10.1533/9780857093134.303 . hal-01008641

\section{HAL Id: hal-01008641 https://hal.science/hal-01008641}

Submitted on 22 Oct 2017

HAL is a multi-disciplinary open access archive for the deposit and dissemination of scientific research documents, whether they are published or not. The documents may come from teaching and research institutions in France or abroad, or from public or private research centers.
L'archive ouverte pluridisciplinaire HAL, est destinée au dépôt et à la diffusion de documents scientifiques de niveau recherche, publiés ou non, émanant des établissements d'enseignement et de recherche français ou étrangers, des laboratoires publics ou privés. 


\title{
OVERALL BEHAVIOUR OF A TRUSS OF FIBRES LINKED BY VISCOUS JOINTS.
}

\author{
S. Le Corre ${ }^{* / * *}$, D. Caillerie ${ }^{*}$, D. Favier ${ }^{*}$, L. Orgéas ${ }^{*}$ \\ ${ }^{*}$ Laboratoire Sols, Solides Structures, \\ CNRS - Univ. Joseph Fourier - Inst. Nat. Polytechnique de Grenoble, France. \\ ${ }^{* *}$ Schneider Electric Industries, \\ C.R.D., Analytics Group, F-38050 Grenoble cedex 9, France.
}

\begin{abstract}
A theoretical method is proposed in order to analyse the behaviour during forming of short fibre reinforced composites. The method applies to high concentrations of fibres where the behaviour is assumed to be dominated by fibre-fibre interactions. Fibres are looked as an assembly of rigid bars linked by viscous joints. The equivalent continuum constitutive relationship is obtained by assuming a periodic spatial repartition of the fibres and applying the method of discrete homogenisation. Theoretical developments lead to two models, a Cosserat continuous medium, or a usual Cauchy one. Lastly a numerical application to planar composites is presented.
\end{abstract}

\section{INTRODUCTION}

In the processing of composites made of a fluid polymeric matrix reinforced by short fibres, fibres noticeably influence the flow of the matrix, and conversely, the flow of the matrix determines the spatial orientation and distribution of fibres. Many theoretical works have been published for low concentrations of fibres. These works intend to deduce the flow of the suspensions from the analysis of the fibre-matrix interaction. The microscopic mechanisms are less known for highly concentrated composites [1]-[3], and simulations of the flow based on their modelling are few and require large computing time [4] even for Newtonian matrices.

A novel approach towards the modelling of such complex problems is presented in this paper. We consider a net of rigid fibres, called bars, linked by linear viscous joints modelling the short-range fibre-fibre interactions. Long range ones are neglected as proposed in [5]. To determine the equivalent continuum behaviour of the assembly, the net is assumed to be periodic. We then use an upscaling method previously developed for quasi-periodic lattice structures in the scope of elasticity [6], [7], [8].

The structure is supposed to be made of a large number $N_{c}$ of identical cells, each filled with $n$ fibres. The size of the structure $L$ is supposed to be large compared to the length of bars $l$, and the ratio $\varepsilon=l / L$ is the small parameter of the problem.

In this paper, two-dimensional assemblies are considered, but the extension to the three dimensional case is straightforward. Geometry, kinematics and local behaviour are first detailed. Then the homogenisation method is developed and we show that different types of constitutive equations are possible. Lastly, an application to planar fibre assemblies is discussed.

\section{DESCRIPTION OF THE GEOMETRY}

The net of fibres is made of bars, linked with the others by several punctual joints. The geometry of the assembly is therefore defined by the spatial and angular position of each fibre, and by the connectivity of each fibre with the remaining of the assembly. The assumption of periodicity suggests a system of numbering of bars and links reflecting the regularity of the microstructure. Cells are located by a vector of integers 
$\underline{a}$ whose components are $\left(a_{1}, a_{2}\right)$. Bars are numbered by a couple $\tilde{b}=(b, \underline{a})$, pointing out the bar $b$ of the cell $\underline{a}$. Similarly, the action of the bar $\tilde{c}$ on the bar $\tilde{b}$ is denoted either by $\tilde{k}=(\tilde{c}, \tilde{b})$ or by $\tilde{k}=(k, \underline{a})$. In the last case, $\tilde{c}$ and $\tilde{b}$ are respectively denoted $[\tilde{k}$ and $\tilde{k}]$. In $\tilde{b}=(b, \underline{a})$ and $\tilde{k}=(k, \underline{a})$, integers $b$ and $k$ number the bars and connections of a reference cell whose corresponding sets are denoted $B$ and $C$, respectively. $\tilde{k}]$ is considered as belonging to the cell $\underline{a}$, thereafter denoted $\underline{a}^{\tilde{k}]}$ or $\underline{a}^{\tilde{b}}$. ${ }^{t} \tilde{k}$ denotes the reciprocal action $(\tilde{b}, \tilde{c})$. The size of cells is assumed to be greater than the length of fibres, $l$. This implies that $\underline{a}^{[\vec{k}}$, the belonging cell of $[\tilde{k}$ is actually a neighbouring cell $\underline{a}^{\tilde{k}]}$. Therefore we introduce $\underline{\delta}^{k}=\underline{a}^{[\tilde{k}}-\underline{a}^{\tilde{k}]}$, vector of integers, whose components take values in $\{-1,0,+1\}$. Because of the geometrical periodicity, $\underline{\delta}^{k}$ is independent of the considered cell.

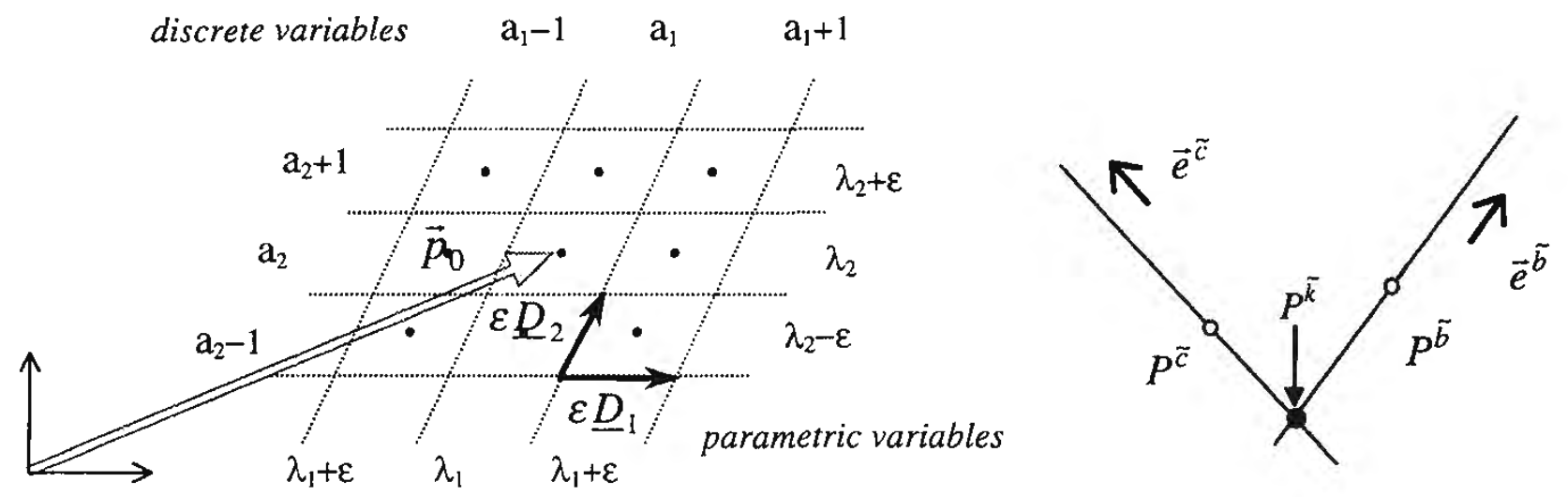

Figure 1 : description of the assembly and numbering system.

Figure 2 : locating of fibres.

Figure 1 sums up the numbering and parameterisation of the cells of the assembly. The position of a bar $\tilde{b}$, see Figure 2 , is defined by the position of its centre $P^{\widetilde{b}}$ located by the vector $\vec{p}(\tilde{b})$, and by its unitary vector $\vec{e}^{\tilde{b}}$. The structure is assumed to be periodic with vectors of periodicity denoted $\varepsilon \vec{D}_{i}$, whose norms are small compared to $L$. Bars centre position are then given by :

$$
\vec{p}(\tilde{b})=\vec{p}(b, \underline{a})=\vec{p}_{0}^{a}+\varepsilon \vec{p}_{1}^{b}=\varepsilon a_{i} \vec{D}_{i}+\varepsilon \vec{p}_{1}^{b}
$$

where $\vec{p}_{0}^{a}$ represents the physical position of the cell inside the structure and $\vec{p}_{1}^{b}$ is the relative position of the bar inside its cell. It is worth noting that $\vec{p}_{1}^{b}$ is independent of the considered cell since the structure is exactly periodic. Introducing the pseudocontinuous variable $\underline{\lambda}=\varepsilon \underline{a}$ one can write :

$$
\vec{p}(\tilde{b})=\lambda_{i} \vec{D}_{i}+\varepsilon \vec{p}_{1}^{b}=\vec{p}_{0}(\underline{\lambda})+\varepsilon \vec{p}_{1}^{b}
$$

So macroscopic positions are parameterised by $\underline{\lambda} \in \Lambda \subset[0,1] \times[0,1] . \Lambda$ is a reference space, where the structure is made of square cells of sides $\varepsilon$. As $\varepsilon$ is assumed to tend to zero, $\underline{\lambda}=\varepsilon \underline{a}$ tends to be continuous, what allows to use it as a continuous variable. 


\section{DESCRIPTION OF MOTION AND INTERACTIONS RELATIONS}

All the fields relative to a bar $\tilde{b}=\tilde{k}$ ] are supposed to have asymptotic expansions of the form [6]:

$$
\vec{u}(\tilde{k}])=\vec{u}_{0}^{k]}(\underline{\lambda})+\varepsilon \vec{u}_{1}^{k]}(\underline{\lambda})+\varepsilon^{2} \vec{u}_{2}^{k]}(\underline{\lambda})+\ldots
$$

$\vec{u}_{n}^{k]}$ are continuous functions of $\underline{\lambda}$ generally depending on the bar $\left.b=k\right]$. The expansion of $\vec{u}(\tilde{c})=\vec{u}\left([\tilde{k})=\vec{u}^{[k}\left(\underline{\lambda}+\varepsilon \underline{\delta}^{k}\right)\right.$ is obtained by using a Taylor expansion [6][8], taking advantage from $\varepsilon \underline{\delta}^{k}<<1$ :

$$
\vec{u}(\tilde{c})=\vec{u}^{[k}\left(\underline{\lambda}+\varepsilon \underline{\delta}^{k}\right)=\vec{u}^{[k}(\underline{\lambda})+\varepsilon \underline{\underline{\nabla}} \vec{u}^{[k}(\underline{\lambda}) \cdot \underline{\delta}^{k}+\ldots
$$

where $\underline{\underline{\nabla}}$ is the gradient operator in the parametric space $\Lambda$.

\section{Expansion of kinematics}

As fibres are considered as rigid bars, their motion can be described by the linear velocity of their centre $\vec{v}^{k]}(\underline{\lambda})$ and by their angular velocity $\vec{\omega}^{k]}(\underline{\lambda})$. To simplify the exposition, we introduce the field $\vec{\phi}^{k]}(\underline{\lambda})=L \vec{\omega}^{k]}(\underline{\lambda})$. The velocity of a point $\mathrm{Q}$ belonging to the bar $\tilde{b}$ is then given by:

$$
\vec{v}^{k]}(Q)=\vec{v}^{k]}(\underline{\lambda})+\vec{\omega}^{k]}(\underline{\lambda}) \wedge \overrightarrow{P^{\tilde{k}]} Q}=\vec{v}^{k]}(\underline{\lambda})+\varepsilon \xi_{Q} \vec{\phi}^{k]}(\underline{\lambda}) \wedge \vec{e}^{k]}
$$

considering that $\left\|\overrightarrow{P^{\bar{k}} Q}\right\|=\xi_{Q} l=\varepsilon \xi_{Q} L . \xi_{Q}$ represents the local position of $Q$ on the bar.

\section{Interaction relations}

The action $\tilde{k}$ of $[\tilde{k}$ on $\tilde{k}]$ is supposed to be punctual and Newtonian : it is parted into a force $\vec{f}^{\tilde{k}}$, proportional to the difference of linear velocities $\overrightarrow{\Delta v} \widetilde{k}^{\tilde{k}}=v^{[\tilde{k}}\left(P^{\tilde{k}}\right)-v^{\tilde{k}]}\left(P^{\tilde{k}}\right)$ at the contacting point $P^{\tilde{k}}$, and a moment relative to $P^{\tilde{k}}$ proportional to the difference of angular velocities $\overrightarrow{\Delta \phi}^{\vec{k}}$. Hence, one can write :

$$
\vec{f}^{\tilde{k}}=\mu^{k} \cdot \overrightarrow{\Delta v}^{\tilde{k}} \quad, \quad \vec{m}^{\tilde{k}}=\beta^{k} \overrightarrow{\Delta \phi}^{\tilde{k}}+\varepsilon \xi^{k} \vec{e}^{\tilde{k}]} \wedge \vec{f}^{\tilde{k}}
$$

where $\vec{m}^{\tilde{k}}$ is the moment relative to $P^{\tilde{k}]}$ the centre of the bar $\left.\tilde{k}\right] . \mu^{k}$ and $\beta^{k}$ are respectively called translational and rotational viscosities. They are dependent on $k$ but independent of the cell.

The expansion of local constitutive equations of the link $\tilde{k}$ is obtained by first expanding the difference of velocities at the contacting point $P^{\vec{k}}$, using (3) and (4):

$$
\overrightarrow{\Delta v}^{\tilde{k}}=\vec{v}^{k}\left(P^{k}, \underline{\lambda}+\varepsilon \underline{\delta}\right)-\vec{v}^{k]}\left(P^{k}, \underline{\lambda}\right)
$$

Identifying each order of $\varepsilon$ leads to (for the sake of simplicity, only the two first orders are presented here) :

$$
\left\{\begin{array}{l}
\overrightarrow{\Delta v}_{0}^{k}=\vec{v}_{0}^{[k}(\underline{\lambda})-\vec{v}_{0}^{k]}(\underline{\lambda}) \\
\overrightarrow{\Delta v}_{1}^{k}=\vec{v}_{1}^{[k}(\underline{\lambda})-\vec{v}_{1}^{k]}(\underline{\lambda})+\underline{\underline{\nabla}} \bar{v}_{0}^{[k}(\underline{\lambda}) \cdot \underline{\delta}^{k}+\xi^{k} \vec{\phi}_{0}^{[k} \wedge \vec{e}^{[k}-\xi^{k} \vec{\phi}_{0}^{k]} \wedge \vec{e}^{k]}
\end{array}\right.
$$

Similarly, the expansion of the difference of angular velocities is : 


$$
\left\{\begin{array}{l}
\overrightarrow{\Delta \phi}_{0}^{k}=\vec{\phi}_{0}^{[k}(\underline{\lambda})-\vec{\phi}_{0}^{k]}(\underline{\lambda}) \\
\overrightarrow{\Delta \phi}_{1}^{k}=\vec{\phi}_{1}^{[k}(\underline{\lambda})-\vec{\phi}_{1}^{k]}(\underline{\lambda})+\underline{\underline{\nabla}} \vec{\phi}_{0}^{[k}(\underline{\lambda}) \cdot \underline{\delta}^{k}
\end{array}\right.
$$

Forces and moments are supposed to have asymptotic expansions of the form (3) :

$$
\left\{\begin{array}{l}
\vec{f}^{\tilde{k}}=\vec{f}_{0}{ }^{k}(\underline{\lambda})+\varepsilon \vec{f}_{1}^{k}(\underline{\lambda})+\varepsilon^{2} \vec{f}_{2}{ }^{k}(\underline{\lambda})+\ldots \\
\vec{m}^{k}=\vec{m}_{0}{ }^{k}(\underline{\lambda})+\varepsilon \vec{m}_{1}{ }^{k}(\underline{\lambda})+\varepsilon^{2} \vec{m}_{2}{ }^{k}(\underline{\lambda})+\ldots
\end{array}\right.
$$

The identification of the terms of their expansions is achieved by introducing (8) and (9) in (10), and assuming that $\beta^{k}=\varepsilon^{q} \beta_{0}^{k}, q$ being a positive integer.

For the forces, the relations are written as :

$$
\vec{f}_{0}^{k}=\mu_{0}^{k} \overrightarrow{\Delta v}_{0}^{k}, \quad \vec{f}_{1}^{k}=\mu_{0}^{k}{\overrightarrow{\Delta v_{1}}}^{k}, \quad \vec{f}_{2}^{k}=\mu_{0}^{k}{\overrightarrow{\Delta v_{2}}}^{k} \ldots
$$

For the moments, three cases have to be distinguished, depending on the value of $q$. This will be discussed in $\S$ "constitutive equations of the equivalent continuum".

\section{EQUILIBRIUM OF THE ASSEMBLY}

Let us now write the equilibrium of each bar $\tilde{b}=\tilde{k}$ ] of the structure, assuming that both inertia and external moments relative to the centres of the bars are negligible :

$$
\forall \tilde{b}, \sum_{\tilde{k} \in C(\tilde{b})} \vec{f}^{\tilde{k}}+\vec{f}^{e x t / \tilde{b}}=0, \quad \sum_{\tilde{k} \in C(\tilde{b})} \vec{m}^{\tilde{k}}=0 .
$$

$C(\tilde{b})$ is the set of links acting on $\tilde{b}$, and $\bar{f}^{\text {ext } / \tilde{b}}$ are the external force applied at the centre of $\tilde{b}$. They are taken such as

$$
\vec{f}^{e x t / \tilde{b}}=\varepsilon^{2} \vec{f}^{\tilde{b}} .
$$

The expansion of the equilibrium equations for all the bars of the assembly is simplified by introducing a virtual power formulation, using two sets of virtual velocity couples $\vec{v}^{*}(\tilde{b})$ and $\vec{\omega}^{*}(\tilde{b})$. This reads :

$$
\forall \vec{v}^{*}(\tilde{b}), \vec{\omega}^{*}(\tilde{b}), \quad \sum_{\tilde{b}} \sum_{\tilde{k} \in C(\tilde{b})}\left(\left(\vec{f}^{\tilde{k}}+\vec{f}^{e x t / \tilde{b}}\right) \cdot \vec{v}^{*}(\tilde{b})+\vec{m}^{\bar{k}} \cdot \vec{\omega}^{*}(\tilde{b})\right)=0
$$

which can be transformed in :

$$
\forall \vec{v}^{*}(\tilde{b}), \vec{\omega}^{*}(\tilde{b}), \quad \sum_{\tilde{k} \in C(\tilde{b})}\left(\vec{f}^{\tilde{k}} \cdot\left(\vec{v}^{*}\left([\tilde{k})-\vec{v}^{*}(\tilde{k}]\right)\right)+\vec{m}^{\tilde{k}} \cdot\left(\vec{\omega}^{*}\left([\tilde{k})-\vec{\omega}^{*}(\tilde{k}]\right)\right)\right)=\sum_{\tilde{b}} \vec{f}^{e x t / \tilde{b}} \cdot \vec{v}^{*}(\tilde{b})
$$

using $\vec{f}^{k}+\vec{f}^{t k}=0$, and $\vec{m}^{k}+\vec{m}^{k}+\varepsilon \bar{L}^{k} \wedge \vec{f}^{k}=0$, where $\vec{L}^{k}$ is the vector $\vec{p}_{1}^{[k}-\vec{p}_{1}^{k]}$.

Sums like $\sum_{\tilde{b}}$ are split in $\sum_{a_{1}, a_{2}} \sum_{b \in B}$ and it can be shown that $\varepsilon^{2} \sum_{a_{1}, a_{2}}$ is approximated by integrals $\int_{\Lambda}$ when $\varepsilon$ tends to zero [8].

\section{Self equilibrium at the order $\varepsilon^{0}$}

The following virtual functions are then taken into (15):

$$
\forall \tilde{b}, \quad \vec{v}^{*}(\tilde{b})=\varepsilon^{2} \alpha(\underline{\lambda}) \vec{v}^{* b}(\underline{\lambda}) \text { and } \vec{\omega}^{*}(\tilde{b})=\varepsilon^{2} \gamma(\underline{\lambda}) \vec{\omega}^{* b}(\underline{\lambda}) .
$$

The self equilibrium at order $\varepsilon^{0}$ is obtained when $\varepsilon$ tends to zero. It can be shown using the method of [8], that $\forall k, \vec{f}_{0}^{k}=0, \vec{m}_{0}^{k}=0$, and $\vec{v}_{0}^{k}=\vec{v}_{0}$. This result is independent of the value of $q$. 


\section{Equilibrium of the equivalent continuum}

Taking advantage of the former analysis, the formulation of the equilibrium of the equivalent continuum is then obtained by taking into (15) smooth virtual macroscopic fields :

$$
\forall \tilde{b} \in B \quad \vec{v}^{*}(\tilde{b})=\vec{v}_{0}^{*}(\underline{\lambda}), \quad \vec{\omega}^{*}(\tilde{b})=\vec{\omega}_{0}^{*}(\underline{\lambda}),
$$

and making $\varepsilon$ tend to zero. This leads to :

$$
\begin{aligned}
& \forall \vec{v}_{0}^{*}, \vec{\omega}_{0}^{*} \\
& \int_{\Lambda} \sum_{k \in C} \vec{f}_{0}^{k} \cdot \underline{\underline{\nabla}} \vec{v}_{0}^{*} \cdot \underline{\delta}^{k}+\underline{\underline{m}}_{0}^{k} \cdot \underline{\underline{\nabla}} \vec{\omega}_{0}^{*} \cdot \underline{\delta}^{k}+\left(\underline{L}^{k} \wedge \vec{f}_{0}^{k}\right) \cdot \vec{\omega}_{0}^{*}=\int_{\Lambda} \vec{f}^{e x t} \cdot \vec{\omega}_{0}^{*}
\end{aligned}
$$

with :

$$
\vec{f}^{e x t}=\sum_{b \in B} \vec{f}^{b} \text {. This explains why external forces were written as assumed in (13). }
$$

The change of variables $\underline{\lambda} \mapsto \vec{p}_{0}(\underline{\lambda})$ enables to rewrite (18) in terms of the physical velocities gradient. Using $\frac{\partial \vec{v}_{0}^{*}}{\partial \lambda_{i}}=\underline{\underline{\nabla}} \vec{v}_{0}^{*} \cdot \vec{D}_{i}$, the formulation can be rewritten as :

$$
\int_{\Omega} \underline{\underline{\sigma}}_{0} \cdot \underline{\underline{\nabla}}_{x} \vec{\nu}_{0}^{*}+\underline{\underline{\kappa}}_{0} \cdot \underline{\underline{\nabla}}_{x} \vec{\omega}_{0}^{*}+\underline{\zeta}_{0} \cdot \vec{\omega}_{0}^{*}=\int_{\Omega} \vec{F}^{e x t} \cdot \vec{v}_{0}^{*}
$$

(19) is the virtual power formulation of a Cosserat medium, whose local equilibrium equation is written as follows :

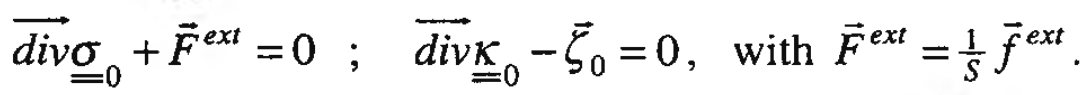

The stress state is determinate by the following three tensors as :

$$
\underline{\underline{\sigma}}_{0}=\frac{1}{S} \sum_{i} \vec{D}_{i} \otimes\left(\underline{\underline{\Sigma}}_{0} \cdot \vec{e}_{i}\right), \quad \underline{\underline{\kappa}}_{0}=\frac{1}{S} \sum_{i} \vec{D}_{i} \otimes\left(\underline{\underline{M}}_{0} \cdot \vec{e}_{i}\right), \quad \vec{\zeta}_{0}=\frac{1}{S} \underline{Z}_{0}
$$

where $S$ is the surface of the reference cell. These tensors are called the stress, the couple-stress, and the first micro-stress tensors, respectively. Their corresponding tensors in the parametric space are :

$$
\underline{\underline{\Sigma}}_{0}=\sum_{k \in C} \vec{f}_{0}{ }^{k} \otimes \underline{\delta}^{k}, \quad \underline{\underline{M}}_{0}=\sum_{k \in C} \vec{m}_{0}{ }^{k} \otimes \underline{\delta}^{k}, \text { and } \quad \underline{Z}_{0}=\sum_{k \in C} \vec{L}^{k} \wedge \vec{f}_{0}{ }^{k} .
$$

\section{CONSTITUTIVE EQUATIONS OF THE EQUIVALENT CONTINUUM}

The last step is now to determine $\vec{f}_{1}^{k}$ and $\vec{m}_{1}^{k}$, in order to obtain the equivalent continuum constitutive relations. This resolution depends of the interactions relations, so three cases have to be distinguished.

Case 1: $q=0$

Interaction laws for forces and moments at the two first orders are

$$
\vec{f}_{1}^{k}=\mu_{0}^{k}{\overrightarrow{\Delta v_{1}}}^{k}, \quad \vec{m}_{1}^{k}=\beta_{0}^{k} \overrightarrow{\Delta \phi}_{1}^{k} .
$$

Carrying into (15) virtual fields of the form:

$$
\forall \tilde{b}, \vec{v}^{*}(\tilde{b})=\varepsilon \alpha(\underline{\lambda}) \vec{v}^{* b}(\underline{\lambda}) \text { and } \vec{\omega}^{*}(\tilde{b})=\varepsilon \gamma(\underline{\lambda}) \bar{\omega}^{* b}(\underline{\lambda}),
$$

and then making $\varepsilon$ tend to zero, on obtains the self-equilibrium of cell at order $\varepsilon^{l}$ :

$$
\forall \vec{v}^{* b}, \sum_{k \in C} \underline{f}_{1}^{k} \cdot\left(\vec{v}^{*[k}-\vec{v}^{* k]}\right)=0, \forall \vec{\omega}^{* b}, \sum_{k \in C} \vec{m}_{1}^{k} \cdot\left(\vec{\omega}^{*[k}-\vec{\omega}^{* k]}\right)=0
$$


In this case, $\vec{m}_{0}^{k}=0$ leads to $\forall k \vec{\phi}_{0}^{k}=\vec{\phi}_{0}$. Both equations are equivalent to linear systems and can be computed using (23) and considering $\underline{\underline{\nabla}} \vec{v}_{0}$ and $\underline{\underline{\nabla}} \vec{\phi}_{0}$ as data. $\underline{\underline{M}} 0$ is non null so that the equivalent continuum is a Cosserat medium. The solution gives $\vec{f}_{1}^{k}$ and $\vec{m}_{1}^{k}$ as linear functions of those gradients, what provides the constitutive equations.

Case $2: q=1$

Interaction laws for forces and moments read :

$$
\vec{f}_{1}^{k}=\mu_{0}^{k} \overrightarrow{\Delta v}_{1}^{k}, \text { and } \vec{m}_{1}^{k}=\beta_{0}^{k} \overrightarrow{\Delta \phi}_{0}^{k}
$$

Using exactly the same process as for Case 2, one obtains the same virtual power formulation of the self-equilibrium (25). Taking into account (26), it can be proved that, once again, $\forall k \vec{\phi}_{0}^{k}=\vec{\phi}_{0}$, what shows that $\forall k \vec{m}_{1}^{k}=0$. The equivalent continuum is therefore a usual Cauchy medium. The solution is computed considering $\underline{\underline{\nabla}} \vec{v}_{0}$ and $\vec{\phi}_{0}$ as data in (25), and also gives the constitutive relations as linear functions in terms of that macroscopic fields.

\section{Case $3: q>1$}

Interaction laws for forces and moments read :

$$
\vec{f}_{1}^{k}=\mu_{0}^{k}{\overrightarrow{\Delta v_{1}}}^{k}, \quad \text { and } \vec{m}_{1}^{k}=0, \quad \vec{m}_{2}^{k}=\beta_{0}^{k} \overrightarrow{\Delta \phi}_{0}^{k}+\mu_{0}^{k} \xi^{k} \vec{e}^{k]} \wedge \overrightarrow{\Delta v}_{1}^{k}
$$

(27) immediately proves that $\forall k \quad \vec{m}_{1}^{k}=0$, so the equivalent continuum is obviously a usual Cauchy one. However, a special formulation has to be employed to solve the problem. Carrying into (15) virtual fields of the form :

$$
\forall \tilde{b}, \vec{v}^{*}(\tilde{b})=\varepsilon \alpha\left(\underline{\lambda} \vec{v}^{*}(\underline{\lambda}) \text { and } \bar{\omega}^{*}(\tilde{b})=\varepsilon \gamma(\underline{\lambda}) \vec{\omega}^{*} b(\underline{\lambda})\right.
$$

and then making $\varepsilon$ tend to zero, on obtains : $\forall \vec{v}^{*}$ and $\vec{\omega}^{*}$

$$
\left.\sum_{k \in C} \underline{f}_{-1}^{k} \cdot\left(\vec{v}^{[k}-\vec{v}^{k}\right]\right)=0 \text { and } \sum_{k \in C} \vec{m}_{2}^{k} \cdot\left(\vec{\omega}^{k[k}-\underline{\vec{\omega}^{* k]}}\right)+\vec{L}^{k} \wedge \vec{f}_{1}^{k} \cdot \vec{\omega}^{*[k}=0 .
$$

which is a special form of self-equilibrium that enables to solve the problem because $\vec{f}_{1}^{k}$ and $\vec{m}_{2}^{k}$ involve the same variables $\vec{v}_{1}^{b}$ and $\vec{\phi}_{0}^{k}$. The solving of (29) is achieved by setting $\underline{\underline{\nabla}} \vec{v}_{0}$ as data and gives $\vec{f}_{1}^{k}$ and $\vec{m}_{2}^{k}$ in terms of it. This formulation leads to a linear constitutive relation linking $\underline{\underline{\sigma}}_{0}$ to $\underline{\underline{\nabla}} \vec{v}_{0}$.

In cases 2 and 3 , the equivalent continuum stress tensor was proved to be symmetrical.

\section{EXAMPLE OF APPLICATION : PLANAR NET}

We shall now present an application of the method to a two-dimensional problem which aims to describe the behaviour of reinforced polymers like GMT or SMC during forming. Those composites are filled with fibre bundles each containing approximately 100 fibres. Bundles are cylinders of length $l$, with an elliptical cross section of minor axis $a$ and major axis $b$. In this work, $b$ is supposed to be oriented in the fibres plane. A dimensional analysis of the interaction relations was led. Approximating the sheared zone between two fibres to a plane of height $\alpha, \beta$ was shown to be of the order $\varepsilon^{4} \mu$. This corresponds to case 3, where local moments are not to be taken into account. For the sake of simplicity, the linear viscosity was taken here such as $\forall k \mu^{k}=\mu$.

The generation of the structure was obtained by a random generation process. This can be achieved quite easily for such planar problems. Positions of bars were defined by two 
spatial and one angular variables that can be simultaneously generated. Intersections of the bars in the 2D plane were then computed. However, some of those intersections do not really exist because the $2 \mathrm{D}$ model neglects the position of bars in the thickness of the material. This method overvalues the number of links. To correct such a default, a statistical analysis was required. This was done by adopting the approach of [10], assuming a square reference cell of sides $l$ and thickness $h$.

In this work, a perfect regularity of the positions of the bars centres was imposed, see Figure 3. Isotropic assemblies were first obtained by randomly choosing the angle of the bar in a set of homogeneously distributed bars. Oriented nets were also generated, assuming a Gaussian repartition of angles with mean 0 , and variance $\theta$. This signifies most fibres are oriented between $-\theta$ and $\theta$. Two examples are presented : oriented 1 is for $\theta=\pi / 4$, oriented 2 for $\theta=\pi / 6$. To characterise the orientation, orientation tensors [11] were used. Theoretically, these orientation tensors should be :

$$
\underline{\underline{A}}_{\text {iso }}=\left[\begin{array}{cc}
0.5 & 0 \\
0 & 0.5
\end{array}\right], \quad \underline{\underline{A}}_{o r i 1}=\left[\begin{array}{cc}
0.682 & 0 \\
0 & 0.318
\end{array}\right], \quad \underline{\underline{A}}_{o r i 2}=\left[\begin{array}{cc}
0.880 & 0 \\
0 & 0.120
\end{array}\right] .
$$

In the presented examples, performed with $l=25 \mathrm{~mm}, h=2 \mathrm{~mm}, \phi=20 \%$, these tensors are :

$$
\underline{A}_{i s o}=\left[\begin{array}{cc}
0.503 & 0 \\
0 & 0.497
\end{array}\right], \quad \underline{A}_{\text {ori }}=\left[\begin{array}{cc}
0.679 & -0.007 \\
-0.007 & 0.321
\end{array}\right], \quad \underline{A}_{\text {ori2 }}=\left[\begin{array}{cc}
0.789 & -0.012 \\
-0.012 & 0.211
\end{array}\right] .
$$

Several preliminary simulations were performed to check that the computed behaviour was actually linear, and that the computed stress tensor was actually symmetrical. The second important test proved that for an isotropic net, the computed response was isotropic. This was proved by submitting the reference cell to the strain rate field :

$$
\underline{\underline{D}}=\frac{1}{2}\left(\underline{\underline{\nabla}} \underline{v}_{0}+{ }^{t} \underline{\underline{\nabla}} \underline{v}_{0}\right) \text { such as } D_{11}=D_{22}=1 \text { and } D_{12}=D_{21}=0
$$

The resulting stresses, shown in Figure $4, \sigma_{11}$ and $\sigma_{22}$ are checked to be almost identical. A first result which shows the interest of our work concerns the analysis of the influence of fibres volume fraction $\phi$. Our simulations, see Figure 4 , show that the viscosity of the net is proportional to $\phi^{2}$, thus proving that it is proportional to the number of connections. The observed scattering of about $\pm 5 \%$ is due to the non perfect generation of isotropic nets. Results perfectly agree with the statistical approach of [10].

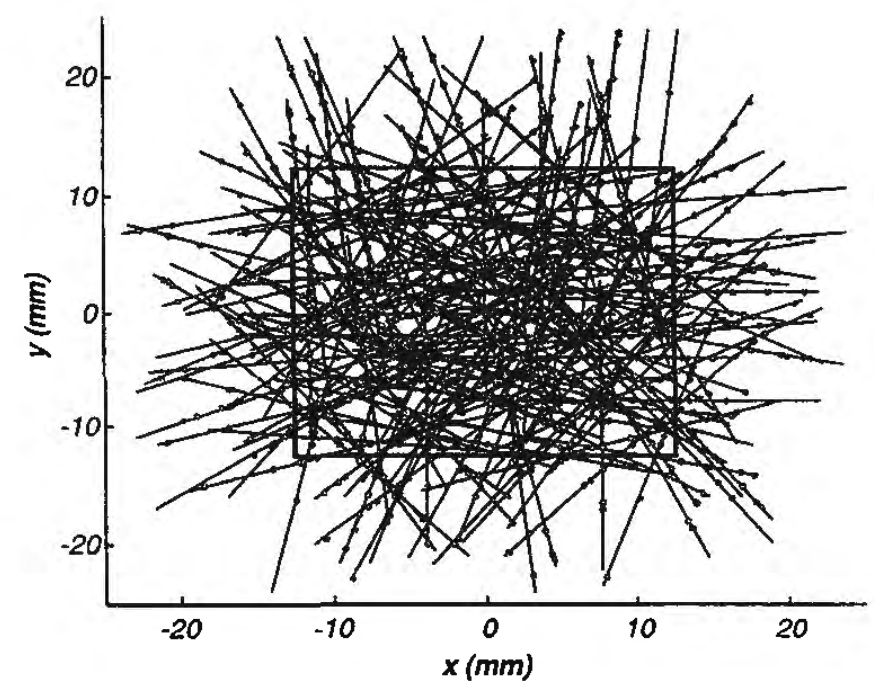

Figure 3 : isotropic generated cell.

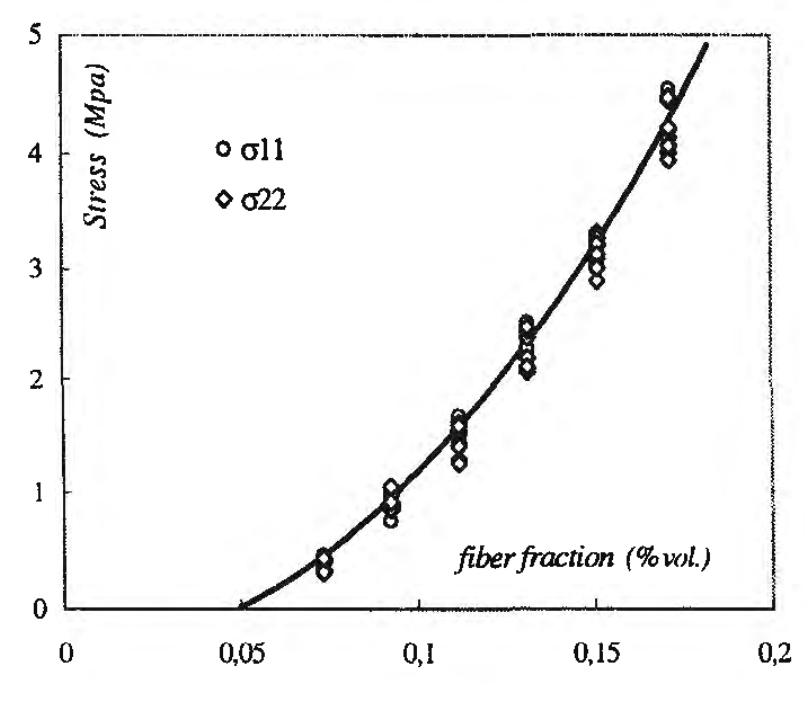

Figure 4: influence of fibre fraction, equibiaxial traction test, isotropic net.

Iso-dissipation curves were plotted in the $\left(\sigma_{11}, \sigma_{22}\right)$ and $\left(D_{11}, D_{22}\right)$ planes, for imposed strain rates such as $D_{12}=D_{21}=0$. And for the three generated assemblies previously presented, results show that fibres orientation strongly modifies the behaviour of the 
net. Identifications of constitutive relations were not carried further. However, results prove that any constitutive relation should use anisotropic functions, and link them to the orientation using a distribution function, or orientation tensors.
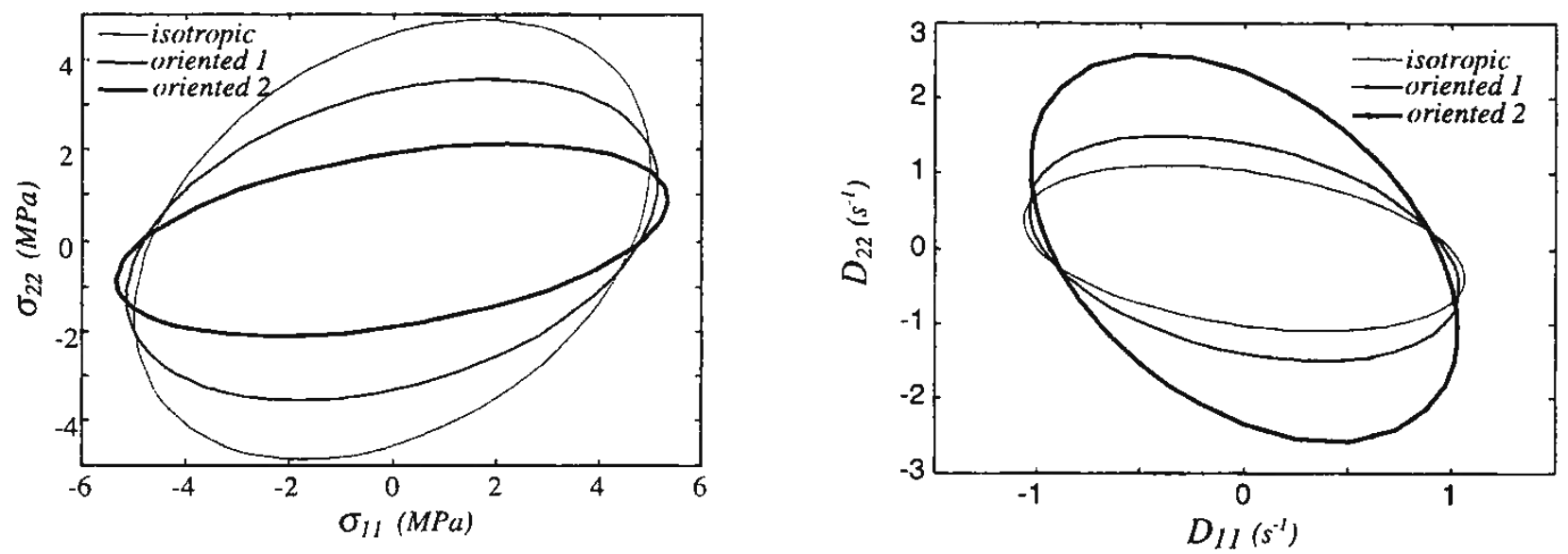

Figure 5: influence of the fibre orientation on the behaviour.

\section{CONCLUSION}

The objective of this work was to present the development of a powerful theoretical tool, whose improvements should bring some interesting answers about the behaviour of fibre suspensions. The method is interesting for it can support any local interaction law, it enables to link the microstructural properties to macroscopic ones, and it is computing time efficient. For the study of fibres suspensions, some extensions are possible such as considering non linear viscous joints and deformable elastic fibres, and taking into account large deformations of the net. This last point would enable to predict the orientation of fibres during forming.

\section{Acknowledgements}

We would like to thank our industrial partners Renault, Renault V.I., Schneider Electric and Inoplast for the financial and technical support they provided to this research. We also thank the council of Région Rhône-Alpes for its financial support.

\section{References}

[1] G.K. Batchelor, "The stress generated in a non-dilute suspension of elongated particles by pure straining motion", J. Fluid Mechanics, 197046 545-570.

[2] F.P. Folgar, C.L. Tucker, "Orientation behavior of fibers in concentrated suspensions". J. Reinforced Plast. Comp., 3,98-119(1984).

[3] E.S.G. Shaqfeh, G.H. Fredricksson, "The hydrodynamic stress in a suspension of rods", Phys. Fluids A, 19902 7-24.

[4] X. Fan, N Phan-Thien, R. Zheng, "Adirect simulation of fibre suspensions". J. Non-Newt. Fluid Mech., 199874 113-135.

[5] Y. Yamane, Y. Kaneda, M. Doi, "Numerical simulation of semi-dilute suspensions of rodlike paricles in shear flow", J. Non-Newt. Fluid Mech., 199454 405-421.

[6] G. Moreau, D. Caillerie, "Continuum modeling of lattice structures in large displacement, application to buckling analysis", Computers and Structures, Special Issue, BHV Topping Ed., 1998 68 181-189.

[7] H. Tollenaere, D. Caillerie, "Continuous modeling of lattice structures by homogenization", Advances in Engineering Softwares, Special Issue, BHV Topping Ed., 1998 29(7/9) 699-705.

[8] G. Moreau, Homogénéisation de structures discrètes en élasticité et en incrémental, PhD Thesis, Institut National Polytechnique de Grenoble, France, 1996.

[9] A. Bensoussan, J.L. Lions, G. Papanicolaou, Asymptotic analysis for periodic structures. North Holland, Amsterdam, 1978.

[10] C. Servais, J-A. E. Manson, S. Toll, "Fiber-fiber interaction in concentrated suspensions : disperse fibers", J. of Rheology, 1999 43(3) 991-1004.

[11] S.G. Advani, C.L. Tucker, "The use of tensors to describe and predict fiber orientation in short fiber composites". J. of Rheology, 1987 31(8) 751-784. 